\title{
Loss of coherence in a system of globally coupled maps
}

\author{
Popovych, O.; Maistrenko, Yu.; Mosekilde, Erik
}

Published in:

Physical Review E. Statistical, Nonlinear, and Soft Matter Physics

Link to article, DOI:

10.1103/PhysRevE.64.026205

Publication date:

2001

Document Version

Publisher's PDF, also known as Version of record

Link back to DTU Orbit

Citation (APA):

Popovych, O., Maistrenko, Y., \& Mosekilde, E. (2001). Loss of coherence in a system of globally coupled maps. Physical Review E. Statistical, Nonlinear, and Soft Matter Physics, 64(2), 026205.

https://doi.org/10.1103/PhysRevE.64.026205

\section{General rights}

Copyright and moral rights for the publications made accessible in the public portal are retained by the authors and/or other copyright owners and it is a condition of accessing publications that users recognise and abide by the legal requirements associated with these rights.

- Users may download and print one copy of any publication from the public portal for the purpose of private study or research.

- You may not further distribute the material or use it for any profit-making activity or commercial gain

- You may freely distribute the URL identifying the publication in the public portal 


\title{
Loss of coherence in a system of globally coupled maps
}

\author{
O. Popovych, ${ }^{1}$ Yu. Maistrenko, ${ }^{1}$ and E. Mosekilde ${ }^{2}$ \\ ${ }^{1}$ Institute of Mathematics, National Academy of Sciences of Ukraine, 01601 Kyiv, Ukraine \\ ${ }^{2}$ Department of Physics, Technical University of Denmark, 2800 Kgs. Lyngby, Denmark
}

(Received 11 August 2000; published 17 July 2001)

\begin{abstract}
We study the formation of symmetric (i.e., equally sized) or nearly symmetric clusters in an ensemble of globally coupled, identical chaotic maps. It is shown that the loss of synchronization for the coherent state and the emergence of subgroups of oscillators with synchronized behavior are two distinct processes, and that the type of behavior that arises after the loss of total synchronization depends sensitively on the dynamics of the individual map. For our system of globally coupled logistic maps, symmetric two-cluster formation is found to proceed through a periodic state associated with the stabilization either of an asynchronous period-2 cycle or of an asynchronous period- 4 cycle. With further reduction of the coupling strength, each of the principal clustering states undergoes additional bifurcations leading to cycles of higher periodicity or to quasiperiodic and chaotic dynamics. If desynchronization of the coherent chaotic state occurs before the formation of stable clusters becomes possible, high-dimensional chaotic motion is observed in the intermediate parameter interval.
\end{abstract}

DOI: 10.1103/PhysRevE.64.026205

PACS number(s): 05.45.Xt

\section{INTRODUCTION}

The purpose of this paper is to study the formation of clusters of partially synchronized behavior in the ensemble

$$
x_{i}(n+1)=(1-\varepsilon) f\left(x_{i}(n)\right)+\frac{\varepsilon}{N} \sum_{j=1}^{N} f\left(x_{j}(n)\right)
$$

of $N$ globally coupled chaotic maps. Here, $i=1, \ldots, N$ is a space index for the $N$-dimensional state vector $\mathbf{x}$ $=\left\{x_{i}(n)\right\}_{i=1}^{N}$, and $n=0,1 \ldots$ is the discrete time variable. $\varepsilon \in \mathbb{R}$ is the coupling parameter and $f: \mathbb{R} \rightarrow \mathbb{R}$ is a onedimensional noninvertible map for which we shall assume the form $f(x)=a x(1-x)$ (the logistic map). $a$ will be referred to as the nonlinearity parameter of the individual map, and the $N$-dimensional map system defined by Eq. (1) will be denoted $\Phi$.

Globally coupled maps of the form (1) were originally introduced by Kaneko [1] in order to study large systems of identical chaotic oscillators interacting via some kind of a mean field. Examples of such systems are typically found in the biological sciences [2]. The insulin producing $\beta$ cells of the pancreas, for instance, are known to display complicated patterns of bursts and spikes in their membrane potentials [3], and these dynamics may also become chaotic. The $\beta$-cells interact with one another through a variety of different mechanisms, including the short range diffusive exchange of ions and small molecules via gap junctions. But there is also a global coupling arising from the response of the whole population of $\beta$-cells to changes in the blood glucose concentrations produced partly through variations in the total release of insulin. As shown by Sturis et al. [4,5], this type of global coupling tends to produce self-sustained oscillations in the insulin secretion with a typical period of 2 hours. Systems of globally coupled chaotic oscillators may also arise in studies of Josephson junction arrays [6] and of multimode lasers [7], and Wang et al. [8] have recently provided experimental evidence of clustering in a system of glo- bally coupled electrochemical reactors. In systems of this type the nonlocal coupling between the chemical reaction sites arises through the electrostatic field; potential changes at some location are rapidly transmitted to other locations.

The simplest form of asymptotic dynamics that can arise in the globally coupled map system (1) is the fully synchronized (or coherent) state in which all elements display the same temporal variation. In this case the motion is restricted to a one-dimensional invariant manifold $D$ $=\left\{\left(x_{1}, x_{2}, \ldots, x_{N}\right) \mid x_{1}=x_{2}=\ldots=x_{N}\right\}$, the main diagonal in $\mathrm{N}$-dimensional phase space, and along this manifold the dynamics is governed by the one-dimensional map $f$ of the individual oscillator. For certain values of the parameters $a$ and $\varepsilon$, the coherent state may attract all trajectories starting from points in its $\mathrm{N}$-dimensional neighborhood. In this case, the coherent state is asymptotically stable.

For other values of $\varepsilon$, the phenomenon of clustering (or partial synchronization) may occur, i.e., the population of oscillators splits into subgroups (clusters) with different dynamics, but such that all oscillators within a given cluster asymptotically move in synchrony. Two-cluster dynamics, for instance, is characterized by a behavior in which two synchronized groups of oscillators are present

$$
\begin{gathered}
x_{i_{1}}=x_{i_{2}}=\cdots=x_{i_{N_{1}}}:=x \\
x_{i_{N_{1}+1}}=x_{i_{N_{1}+2}}=\cdots=x_{i_{N}}:=y,
\end{gathered}
$$

where $N_{1}<N$ and $N_{2}=N-N_{1}$ denote the number of synchronized elements in each of the two clusters.

Under these conditions the $N$-dimensional coupled map system (1) reduces to a system of two coupled onedimensional maps

$$
F:\left(\begin{array}{l}
x \\
y
\end{array}\right) \rightarrow\left(\begin{array}{c}
f(x)+p \varepsilon[f(y)-f(x)] \\
f(y)+(1-p) \varepsilon[f(x)-f(y)]
\end{array}\right),
$$


where the parameter $p$ describes the relative distribution of oscillators between the two clusters. More precisely, $p$ $=N_{2} / N$ denotes the fraction of the total population that synchronizes in state $y$. For $N=3$, for instance, with two clusters $x_{1}=x_{2}:=x$ and $x_{3}:=y$, the dynamics of Eq. (1) is described by the two-dimensional map $F$ with $p=1 / 3$. Clearly, for $N$ $=3$, two-cluster dynamics can be realized in $3 ! /(2 ! 1 !)=3$ different ways. Hence, we have three distinct (and mutually symmetric) two-cluster states. For larger values of $N$, the possible realizations of a given cluster distribution grow very rapidly. In the present paper we shall focus on the formation of symmetric (i.e., equally sized) or nearly symmetric clusters.

In his original work, Kaneko [1] developed a rough phase diagram for the occurrence of different clustering states in the globally coupled map system (1). If the coupling parameter $\varepsilon$ is high enough ( $\varepsilon \gtrsim 0.355$ for $a=3.8$ ), the state of full synchronization attracts almost all trajectories within a large region. For lower values of $\varepsilon$, the coherent state breaks up into a number of clusters. Immediately below the coherent state one typically finds an ordered state with two-cluster dynamics, or, for higher values of $a$, a so-called glassy phase where a few large clusters appear to coexist with many small clusters. Finally, as the coupling parameter becomes small enough, a transition to a turbulent state takes place. Here, almost all attracting states involve a large number of clusters, and the oscillators are nearly completely desynchronized.

In subsequent works, Kaneko has applied the globally coupled map approach as a model of biological cell differentiation [9]. He has also studied the occurrence of Milnor attractors and the role of noise-induced selection in highdimensional systems [10]. Referring to the original definition [11], a Milnor attractor is a state that attracts a positive Lebesgue measure set of points from its neighborhood, but for which this neighborhood may also contain a positive Lebesgue measure set of points that are repelled from the (weakly) attracting state. The existence of such weak attractors is closely linked to the recently discovered phenomena of riddled basins of attraction $[12,13]$ and on-off intermittency [14].

Kaneko's work has also inspired a considerable number of other investigators. In particular, $\mathrm{Xie}$ and $\mathrm{Hu}$ [15] have pointed out that with positive values of the coupling parameter synchronous periodic cycles that are stable for the individual map are also stable in $N$-dimensional phase space. Hence, these authors have discussed the transverse destabilization of periodic orbits in the period-doubling cascade and in the main periodic windows for negative values of the coupling parameter. Glendinning [16] has investigated the fractal nature of the blowout bifurcation in which the coherent state loses its average stability in the transverse direction, illustrating how globally coupled map systems can proceed through a complicated sequence of synchronizations and desynchronizations in connection with transitions between periodic and chaotic dynamics for the individual map.

Considering a system of two coupled, identical logistic maps, Maistrenko et al. [17] have performed a detailed investigation of the so-called riddling bifurcation $[18,19]$ in which the first transverse destabilization of a periodic orbit embedded in the synchronized chaotic state takes place. As illustrated by Eq. (3), the same model can be used to study the transition to two-cluster dynamics for a system of $N$ coupled logistic maps, provided that the maps distribute themselves symmetrically between the two clusters. In a subsequent study by Popovich et al. [20], emphasis was given to the role of an asymmetric distribution of the oscillators. Whereas the transverse period-doubling bifurcation remains essentially unaffected by such an asymmetry, the transverse pitchfork bifurcation was found to be replaced by a transcritical riddling bifurcation in which a periodic orbit born in a saddle-node bifurcation passes through the synchronization manifold and exchanges its transverse stability with a saddle cycle of similar periodicity in that manifold.

Most recently, partial synchronization (or cluster formation) has been studied by Maistrenko et al. [21] in a system of three coupled skew-tent maps and by Taborov et al. [22] in a system of three coupled logistic maps. Applying a special coupling scheme of relevance in connection with applications for secure communication, they have determined the regions in parameter space where total and partial synchronization take place and they have analyzed the bifurcations through which the coherent state (total synchronization) breaks down to give way for two- and three-cluster dynamics.

Hamm [23] has considered the asymptotic behavior of a globally coupled map system in the thermodynamic limit $N$ $\rightarrow \infty$, and Ouchi and Kaneko [24], and Belykh et al. [25] have started to study models with both local and global coupling as a way of understanding hierarchical pattern formation in systems with interactions on different length scales. In this connection it is worth noticing that globally coupled systems differ qualitatively from locally coupled systems with respect to the types of dynamics that they can support. Moreover, in contrast to locally coupled systems, globally coupled systems do not seem to relax towards a statistical equilibrium [26].

With the aim of answering a number of questions that arise in connection with the phase diagram provided by Kaneko [1], the present paper performs a bifurcation analysis of the transition from coherent behavior to two- and threecluster dynamics for the globally coupled map system (1). As previously mentioned we restrict our attention to the emergence of partially synchronized dynamics with an even or nearly even distribution of maps between the clusters. The formation of strongly asymmetric clusters $(p \ll 1)$ constitutes a separate problem with a variety of interesting phenomena. We start in Sec. II by establishing the conditions for transverse stability of attractors belonging to any $K$-dimensional state of the $N$-dimensional map. In Sec. III, a detailed phase diagram is presented. We find that, under proper conditions desynchronization of the coherent state can directly give birth to high-dimensional chaotic dynamics. We suppose that this high-dimensional state has the full dimension of $\mathrm{N}$-dimensional phase space. Finally, Sec. IV is devoted to a study of the stability of chaotic two- and three-cluster states. Here, our computer calculations recover the spurious phenomenon known as synchronization with positive conditional (i.e., transverse) Lyapunov exponents [27,28]. This 
type of numerically generated clustering can lead to false conclusions concerning the occurrence of low-dimensional dynamics. The phenomenon can be removed by adding a small amount of noise to the computations.

\section{STABILITY OF $K$-CLUSTER STATES}

Let us suppose that system (1) falls into a $K$-cluster state, i.e., the coordinates of the state vector $\mathbf{x}=\left\{x_{i}\right\}_{i=1}^{N}$ split into $K$ groups such that in each group the coordinates are identically the same

$$
\begin{gathered}
x_{i_{1}}=x_{i_{2}}=\cdots=x_{i_{N_{1}}}:=y_{1}, \\
x_{i_{N_{1}+1}}=x_{i_{N_{1}+2}}=\cdots=x_{i_{N_{1}+N_{2}}}:=y_{2}, \\
\vdots \\
x_{i_{N_{1}+N_{2}+\cdots+N_{K-1}+1}=}=x_{i_{N_{1}+N_{2}}+\cdots+N_{K-1}+2}=\cdots=x_{i_{N}}:=y_{K} .
\end{gathered}
$$

The positive integer $N_{j}$ represents the number of variables $x_{i}$ belonging to the $j$ th cluster, $j=1,2, \ldots, K$, so that $N_{1}+N_{2}$ $+\cdots+N_{K}=N$. We note that, by virtue of the complete symmetry of the system (i.e., the fact that all the individual maps are the same), for any set $\left\{N_{j}\right\}$ the $K$-dimensional subspace defined by Eqs. (4) remains invariant for the dynamics in the corresponding $K$-cluster state.

Introducing the set of parameters $p_{j}=N_{j} / N, j$ $=1,2, \ldots, K$, the dynamics in the $K$-cluster state can be described by the system of $K$ coupled one-dimensional maps

$$
\begin{array}{r}
y_{i}(n+1)=(1-\varepsilon) f\left(y_{i}(n)\right)+\varepsilon \sum_{j=1}^{K} p_{j} f\left(y_{j}(n)\right), \\
i=1, \ldots, K .
\end{array}
$$

This system is also a globally coupled map system, but with different weights $p_{j}$ associated with the contribution of the $j$ th cluster to the global coupling. Varying the parameters $p_{j}$ in Eq. (5) we can obtain the governing map for any possible $K$-cluster dynamics of our original system (1).

A necessary condition for the presence of stable $K$-cluster behavior in system (1) is that the map (5) with the assumed values of the parameters $p_{j}$ has a stable invariant set $A^{(K)}$, but that there is no stable invariant sets $A^{(L)}$ with $L<K$. For example, system (1) with even number of cites $N$ may demonstrate symmetric two-cluster dynamics (2) if the twodimensional map (3) with $p=0.5$ has a stable invariant set $A^{(2)} \nsubseteq D=\{(x, y) \mid x=y\}$.

Provided that it is stable in the cluster subspace, the conditions for an attractor $A^{(K)}$ of system (5) to be stable in the whole $N$-dimensional phase space are that it is also stable in the transverse directions. The transverse stability of $A^{(K)}$ may be asymptotic, when it attracts all trajectories from its neighborhood, or weak, when $A^{(K)}$ is stable in the Milnor sense, i.e., it attracts a positive Lebesgue measure set of initial data [11].
In order to examine the conditions for transverse stability of the two-cluster state (2) we consider the Jacobian matrix $D F$ of the $N$-dimensional map $\Phi$ defined by Eq. (1). Reduced on the subspace defined by Eqs. (2), the matrix $D F$ can be represented as

$$
D \Phi=\left[\begin{array}{ll}
M(x) & L(y) \\
L^{T}(x) & N(y)
\end{array}\right],
$$

where $M(x)$ and $N(y)$ are symmetric matrices of dimensions $N_{1} \times N_{1}$ and $N_{2} \times N_{2}$, respectively, and $T$ denotes the operation of transposition. It is easy to show that the matrix $D \Phi$ has two distinct eigenvalues $\nu_{\perp, 1}=f^{\prime}(x)(1-\varepsilon)$ and $\nu_{\perp, 2}$ $=f^{\prime}(y)(1-\varepsilon)$ that occur with the multiplicities $N_{1}-1$ and $N_{2}-1$, respectively.

Let now the two-dimensional map (2) have an attractor $A^{(2)}$ that does not belong to the diagonal $D=\{(x, y) \mid x=y\}$. By virtue of the form of the transverse eigenvalues $\nu_{\perp, 1,2}$ and the fact that the corresponding eigenvectors do not depend on the phase coordinates, the transverse Lyapunov exponents of the two-cluster state are given by

$$
\begin{aligned}
\lambda_{\perp, 1}^{(2)} & =\lim _{k \rightarrow \infty} \frac{1}{k} \sum_{n=0}^{k-1} \ln \left|f^{\prime}(x(n))(1-\varepsilon)\right| \\
& =\lim _{k \rightarrow \infty} \frac{1}{k} \sum_{n=0}^{k-1} \ln \left|f^{\prime}(x(n))\right|+\ln |1-\varepsilon|, \\
\lambda_{\perp, 2}^{(2)} & =\lim _{k \rightarrow \infty} \frac{1}{k} \sum_{n=0}^{k-1} \ln \left|f^{\prime}(y(n))(1-\varepsilon)\right| \\
& =\lim _{k \rightarrow \infty} \frac{1}{k} \sum_{n=0}^{k-1} \ln \left|f^{\prime}(y(n))\right|+\ln |1-\varepsilon|,
\end{aligned}
$$

evaluated for a typical trajectory $\{(x(n), y(n))\}_{n=0}^{\infty} \subset A^{(2)}$.

As discussed above, the attractor $A^{(2)}$ for the system (3) of two-coupled maps is at least a Milnor attractor for the $\mathrm{N}$-dimensional system (1) when it attracts a positive Lebesgue measure set of points from $R^{N}$. For this to occur, both the above Lyapunov exponents must be negative [18]. Hence, a procedure for finding stable two-cluster states in system (1) can be the following. First, we find an attractor $A^{(2)} \nsubseteq D=\{(x, y) \mid x=y\}$ for the system of two coupled maps (3). Then two Lyapunov exponents $\lambda_{\perp, i}, i=1,2$ of the form (6) are calculated for typical trajectories on $A^{(2)}$. For the parameter region where both of these Lyapunov exponents are negative, the system of globally coupled maps (1) has a stable (at least in average) two-cluster state with a dynamics given by the two-cluster attractor $A^{(2)}$. This procedure does not depend on the number $N$ of coupled oscillators in Eq. (1). The only restriction is that this number should allow the assumed distribution of variables between the clusters. For example, if the two-dimensional system (3) with $p=1 / 3$ has an attractor $A^{(2)}$ (not belonging to the diagonal $D$ ), and both the transverse Lyapunov exponents are negative, then the $\mathrm{N}$-dimensional system (1) will have corresponding stable 
two-cluster states at $N=3\left(N_{1}=2, N_{2}=1\right), N=6\left(N_{1}=4, N_{2}\right.$ $=2), N=9\left(N_{1}=6, N_{2}=3\right), N=12\left(N_{1}=8, N_{2}=4\right)$, etc.

In the case of periodic dynamics, it can be shown that if the two-dimensional map $F$ with $0 \leqslant \varepsilon \leqslant 1$ has a stable period- $2^{k}, k=1,2, \ldots$, cycle out of the diagonal with symmetric distributions of its points with respect to the diagonal (e.g., for $p=0.5$ ) then system (1) exhibits stable period- $2^{k}$ two-cluster dynamics.

By analogy with the two-cluster state, for a $K$-cluster state (4) with the attractor $A^{(K)}$, one has to iterate the map (5) on $A^{(K)}$ and calculate $K$ transverse Lyapunov exponents as given by

$$
\begin{gathered}
\lambda_{\perp, j}^{(K)}=\lim _{k \rightarrow \infty} \frac{1}{k} \sum_{n=0}^{k-1} \ln \left|f^{\prime}\left(y_{j}(n)\right)\right|+\ln |1-\varepsilon|, \\
j=1,2, \ldots, K .
\end{gathered}
$$

When all the Lyapunov exponents are negative, $A^{(K)}$ is also an attractor in $N$ dimensions in the Milnor sense [11]. This provides the conditions for the existence of stable $K$-cluster states for system (1).

\section{DIFFERENT WAYS OF DESYNCHRONIZATION FOR THE COHERENT STATE}

The purpose of this section is to discuss Kaneko's phase diagram [1] for the occurrence of the various clustering states in more detail and to identify the different types of bifurcations that occur as the coupling constant $\varepsilon$ and the nonlinearity parameter $a$ are varied.

Coherent motion of the coupled map system (1) takes place on the main diagonal $D=\left\{\left(x_{1}, x_{2}, \ldots, x_{N}\right) \mid x_{1}=x_{2}\right.$ $\left.=\cdots=x_{N}\right\}$ of the $N$-dimensional phase space and is governed by the logistic map $f=f_{a}$. Depending on the value of $a$, the coherent dynamics may be either periodic or chaotic, as characterized by the sign of the Lyapunov exponent

$$
\lambda_{a}=\lim _{k \rightarrow \infty} \frac{1}{k} \sum_{n=0}^{k-1} \ln \left|f^{\prime}(x(n))\right|
$$

calculated for a typical trajectory $\{x(n)\}_{n=0}^{\infty}$ of $f_{a}$. For an ensemble of coupled logistic maps this implies that, for any particular value of $a$, only a single one-dimensional attractor, periodic or chaotic, can exist on $D$. Let us denote it by $A^{(s)}$, where the superscript denotes "symmetric."

The average transverse stability of the attractor $A^{(s)}$ is determined by the transverse Lyapunov exponent $\lambda_{\perp}^{(1)}=\lambda_{a}$ $+\ln |1-\varepsilon|$. Actually, there are $N-1$ transverse Lyapunov exponents but, due to the symmetry of system (1), they are all equal to $\lambda_{\perp}^{(1)}$ (see Sec. II) . Hence, the coherent motion loses its transverse stability simultaneously in all $N-1$ independent transverse directions.

\section{A. Two steps of desynchronization: Riddling and blowout}

Transverse destabilization of the coherent motions, if they are chaotic, takes place in two steps. First, the chaotic attractor $A^{(s)} \subset D$ loses its asymptotic transverse stability in a rid-

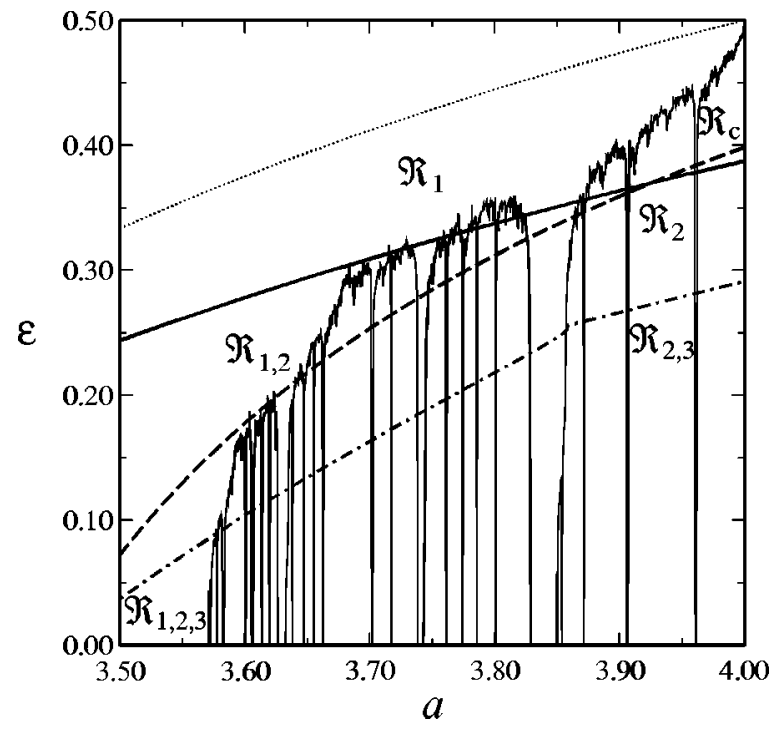

FIG. 1. Phase diagram for cluster formation in a system of globally coupled logistic maps. $a$ is the nonlinearity parameter for the individual map, and $\varepsilon$ is the coupling parameter. The uppermost (dotted) curve represents the riddling bifurcation of the one-piece chaotic coherent state $A^{(s)}$ in which the fixed point $P_{1}^{(s)} \in A^{(s)}$ loses its transverse stability, and the fully drawn fractal curve delineates the blowout bifurcation. The smooth fully drawn and dashed bold curves represent stabilization of the asynchronous period-2 and period-4 cycles in the symmetric two-cluster states, respectively. The lowermost (dashed-dotted) curve represents the stabilization of (another) period-4 cycle in the symmetric three-cluster state. Regions denoted by $\mathfrak{R}$ correspond to parameter values where the system has stable clusters, and subscripts indicate the cluster numbers. $\mathfrak{R}_{c}$ denotes the region where the dynamics is high-dimensional chaotic.

dling bifurcation $[18,19]$. This occurs when the first trajectory embedded in the synchronous chaotic state becomes transversely unstable. After the riddling bifurcation, $A^{(s)}$ is no longer stable in the Lyapunov sense. In any small neighborhood of the attractor one can find a positive measure set of phase points such that the trajectories, when starting from these points, will go away from $A^{(s)}$. Provided that other asymptotic states, which can be reached from the neighborhood of $A^{(s)}$, do not exist, most of the trajectories will sooner or later return to the neighborhood of $A^{(s)}$. In the presence of noise, some of the trajectories may again perform a burst, manifesting the typical bubbling behavior [18]. This type of characteristic phase dynamics is associated with the weak (or Milnor) stability of $A^{(s)}$. It gives rise to locally riddled basins of attraction for the synchronous chaotic state $[18,19]$.

In the phase diagram of Fig. 1, the uppermost (dotted) curve denotes the transverse destabilization of the fixed point $P_{1}^{(s)}=\left(x_{0}, x_{0}, \ldots, x_{0}\right), x_{0}=1-1 / a$. In the parameter $a$ regime $\left(a>a_{0} \cong 3.678573\right)$ where $A^{(s)}$ is one-piece chaotic, $P_{1}^{(s)}$ is the first trajectory on $A^{(s)}$ to lose its transverse stability, and, hence, the dotted curve represents the riddling bifurcation curve. This curve can easily be determined analytically [17]. Below the riddling bifurcation curve the coherent chaotic state is weakly stable only. Destabilization of $P_{1}^{(s)}$ takes place via a transverse period-doubling bifurcation and 
produces an asynchronous period-2 saddle around the fixed point. For slightly lower values of the coupling parameter, the synchronous period- 2 cycle embedded in the coherent chaotic state also undergoes a transverse period-doubling, producing an asynchronous period-4 cycle.

The fractal curve in Fig. 1 denotes the blowout bifurcation of $A^{(s)}$. The blowout occurs at $\varepsilon=\varepsilon_{b l}=1-e^{-\lambda_{a}}$ when the transverse Lyapunov exponent $\lambda_{\perp}^{(1)}$ of the synchronous chaotic set changes its sign from minus to plus. After the blowout bifurcation, $A^{(s)}$ is no longer an attractor but has turned into a chaotic saddle. Almost all trajectories now go away from the coherent state described by the chaotic set $A^{(s)}$, and in general only a zero measure set of trajectories will approach $A^{(s)}$ [18]. One of the main questions of the present paper is to determine the fate of the diverging trajectories. We find that, depending sensitively on $a$, there are two different possibilities associated with the mutual disposition of the blowout and two-cluster stabilization curves.

Let $a$ be fixed and let us consider what happens as the control parameter $\varepsilon$ is reduced. If the blowout bifurcation occurs before the appearance of a stable two-cluster state, the coherent phase turns into a high-dimensional chaotic state. With further reduction of parameter $\varepsilon$, this may be captured into one of the periodic two-cluster states. In the opposite situation, i.e., when the asynchronous periodic cycles stabilize before the blowout bifurcation, two-cluster states appear before the blowout of the coherent state. As a consequence, both types of dynamics-fully synchronized chaotic and two-cluster periodic-coexist in some region of the $(a, \varepsilon)$-parameter plane [20].

In Fig. 1, the solid and dashed bold curves represent the stabilization of the asynchronous cycles $P_{2}$ (period-2) and $P_{4}$ (period-4) forming the possible symmetric (or close to symmetric) two-cluster states. These cycles remain stable in some regions under the curves to destabilize with further reduction of $\varepsilon$ in a Hopf bifurcation. The symmetric twocluster state $P_{2}$, which arises as the asynchronous saddle cycle produced through a transverse period-doubling bifurcation of the symmetric fixed point $P_{1}^{(s)}$, stabilizes in a subcritical, inverse pitchfork bifurcation along the fully drawn bold curve. $P_{4}$, which arises from a transverse period doubling of the symmetric period-2 orbit, stabilizes along the dashed bold curve. It can be seen in Fig. 1 that, for $a$ $\geq 3.93, P_{4}$ stabilizes before (i.e. for higher values of $\varepsilon$ than) $P_{2}$. Moreover, slightly asymmetric two-cluster states stabilize after the symmetric ones when $\varepsilon$ decreases. In Sec. III C we shall perform a more detailed analysis of the influence of cluster asymmetry on the stabilization of the cycles $P_{2}$ and $P_{4}$ (and the dynamics developed from these cycles). The idea is to illustrate the important role played by the exactly symmetric two-cluster states for the synchronization phenomena in system (1).

The last (dotted-dashed) bifurcation curve shown in Fig. 1 represents the stabilization of the symmetric three-cluster state. In the moment of this bifurcation, stable period-4 cycles appear in each of the subspaces for the symmetric three-cluster states whose dynamics is governed by system (5) with $K=3$ and $p_{j}=1 / 3, j=1,2,3$. In the region of inter- est this three-cluster curve lies below the two-cluster curve given by the stabilization of $P_{2}$ and $P_{4}$. Therefore, we assume that the two-cluster bifurcation curve delineates the first moment of formation of symmetric clusters in the globally coupled map system (1).

\section{B. Dynamics in the two-cluster state}

In the two-cluster states, the dynamics is governed by the two-dimensional map (3). Figure 2(a) shows a characteristic phase portrait after the riddling bifurcation. The fixed point $P_{1}^{(s)}=\left\{x_{0}=y_{0}=1-(1 / a)\right\}$ belonging $A^{(s)}$ has become transversely unstable in a period-doubling bifurcation giving rise to a saddle period-2 cycle $P_{2}$. The thin curves connecting $P_{1}^{(s)}$ with the points of $P_{2}$ represent a separatrix. Close to this separatrix the trajectories will first approach $P_{2}$ and then proceed along one of the unstable manifolds of the saddle cycle. Hence, there exists a positive measure set of the trajectories that, when starting near $P_{1}^{(s)}$, can move away from $A^{(s)}$ to a distance given by the deviation of $P_{2}$ from $P_{1}^{(s)}$. As the preimages of the fixed point $P_{1}^{(s)}$ are dense in $A^{(s)}$, we conclude that in the neighborhood of any point of $A^{(s)}$, there exists a positive measure set of points that give rise to trajectories that go away from $A^{(s)}$ in the direction towards $P_{2}$, i.e., the basin of attraction of $A^{(s)}$ is locally riddled.

Trajectories that burst away from $A^{(s)}$ are restricted to an absorbing area denoted in Fig. 2(a) by $\mathcal{A}$. This invariant region is bounded by the segments of the critical curves $L_{1}$ and $L_{2}[29,30]$. These curves are obtained as the first and the second images of the set

$$
L_{0}=\left\{(x, y) \mid\left(x-\frac{1}{2}\right)\left(y-\frac{1}{2}\right)=0\right\},
$$

which is the locus of points in $\mathbb{R}^{2}$ where the Jacobian $D F$ of the map $F$ in Eq. (3) vanishes. As long as the basin of $A^{(s)}$ is locally riddled only (no other attractors inside $\mathcal{A}$ ), most of the trajectories entering into bursts will eventually be attracted by $A^{(s)}$.

As we can see from Fig. 1, this type of locally riddled dynamics occurs for a relatively wide region (denoted $\mathfrak{R}_{1}$ ) of the $(a, \varepsilon)$-parameter plane. The lower boundary of this region consists of two very different parts: a fractal boundary defined by the blowout bifurcation curve, and a smooth boundary corresponding to the symmetric two-cluster formation curve. The corresponding transformations of the dynamics of the system clearly involve very different processes.

If the parameter point $(a, \varepsilon)$ leaves the region $\Re_{1}$ through the fractal (blowout) curve, the absorbing area $\mathcal{A}$ defines a new attractor in the plane of the two-cluster state. This is illustrated in Fig. 2(b). As we shall see in Sec. IV, however, this type of two-dimensional attractor arising from the coherent state in a blowout bifurcation is not stable in the whole $N$-dimensional phase space. Transverse to the two-cluster state, the maximal Lyapunov exponent $\lambda_{\perp}^{(2)}$ is positive although small, growing according to the power law $\mid \varepsilon$ $-\left.\varepsilon_{b l}\right|^{\alpha}, 1 \leqslant \alpha \leqslant 2$, where $\varepsilon_{b l}$ is the blowout bifurcation value.

Consider now in more detail the second possibility where the $(a, \varepsilon)$-parameter point leaves $\mathfrak{R}_{1}$ through the smooth 

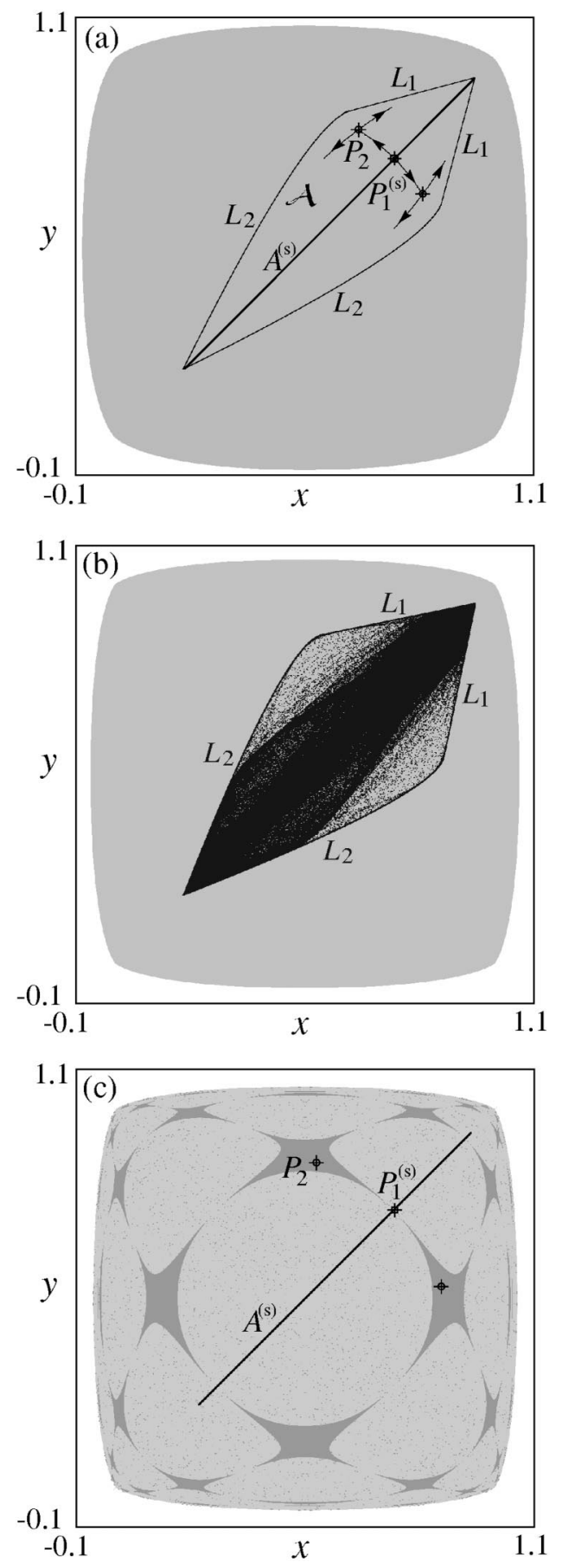

FIG. 2. Typical phase portraits for the globally coupled map system (1) reduced to the symmetric two-cluster subspace $[p=0.5$ in Eq. (3)]: (a) locally riddled basin of attraction for the coherent state $A^{(s)}$ after the riddling bifurcation ( $a=3.8, \varepsilon=0.42$ ), (b) on-off intermittency after the blowout bifurcation of $A^{(s)}(a=3.8, \varepsilon$ $=0.34$ ), and (c) globally riddled basin of attraction for $A^{(s)}$ after stabilization of the asynchronous period-2 cycle $P_{2}(a=3.75, \varepsilon$ $=0.315$ ). The light gray region in (a) and (c) represents the basin of attraction for $A^{(s)}$, and the basin of attraction for the on-off attractor in (b). The dark gray regions in (c) represent the basin of attraction for the cycle $P_{2}$ whose points are plotted by crossed circles. The curves $L_{1}$ and $L_{2}$ delineate the absorbing area $\mathcal{A}$, and $P_{1}^{(s)}$ is the fixed point embedded in $A^{(s)}$. Note that the on-off state in (b) is not stable in $N$-dimensional phase space. two-cluster formation curve. The characteristic phase portrait after this transition is presented in Fig. 2(c). Two different asymptotic states coexist: a coherent state given by the synchronous chaotic set $A^{(s)}$ and a periodic two-cluster state given by the cycle $P_{2}$. The basin of attraction for the coherent attractor becomes globally riddled with the basin of $P_{2}$. In the phase diagram of Fig. 1, the parameter region where this kind of globally riddled dynamics occur, is denoted by $\mathfrak{R}_{1,2}$. When $(a, \varepsilon)$ belongs to $\mathfrak{R}_{1,2}$, both coherent and twocluster regimes can be realized in system (1) when calculations are performed with randomly chosen initial conditions. This follows from the stability of the cycles in $N$ dimensions as soon as they are stable in the two-cluster state (see Sec. II).

The lower boundary of $\Re_{1,2}$ in Fig. 1 is given by the blowout bifurcation curve of the coherent attractor $A^{(s)}$. Under this curve, $A^{(s)}$ is no longer stable even in average. Hence, only two-cluster stable regimes can be manifested in system (1) provided that the parameter point $(a, \varepsilon)$ lies above the three-cluster dotted-dashed curve ( $\Re_{2}$ region). Below the latter curve two- and three-cluster states coexist ( $\mathfrak{R}_{2,3}$ region). Moreover, in the lower left corner of Fig. 1, one can observe a parameter region where the blowout curve falls below the three-cluster curve ( $\mathfrak{R}_{1,2,3}$ region). Here, the coherent chaotic state and the two-cluster state coexist with three-cluster dynamics.

The last region in Fig. 1, denoted by $\mathfrak{R}_{c}$, is bounded by the blowout curve from above and by the symmetric twocluster formation curves from below. Here, the dynamics of system (1) can be high-dimensional chaotic, provided that strongly asymmetric clusters do not arise. We justify this statement in Sec. IV by showing that symmetric two- and three-cluster states are unstable in the whole $N$-dimensional phase space of system (1) and that the dynamics in $\Re_{c}$ may be completely uncorrelated, i.e., it is not attracted by a cluster state of lower dimension. The role of strongly asymmetric clusters for the formation of partially synchronized states will be considered in a forthcoming publication [31].

\section{Formation of the symmetric clusters}

As shown above, the appearance of the symmetric (or slightly asymmetric) two-cluster dynamics in the globally coupled map system (1) is caused by the stabilization of the period- 2 or period-4 asynchronous cycles $P_{2}$ and $P_{4}$. In this section we shall consider how the moments of stabilization depend on a small cluster asymmetry, i.e., when the parameter $p$ in system (3) starts to differ from 0.5. A main conclusion is that the symmetric clusters, i.e., with $p=0.5$, stabilize at higher values of the coupling parameter $\varepsilon$ than other, slightly asymmetric clusters. Moreover, the later the stabilization occurs the larger the asymmetry is. For the symmetric two-cluster state $(p=0.5)$, the cycles $P_{2}$ and $P_{4}$ are born in transverse period-doubling bifurcations of the coherent fixed point $P_{1}^{(s)}$ and $P_{2}^{(s)}$, respectively. After the bifurcations they are first unstable (saddles) to later stabilize in inverse subcritical pitchfork bifurcations. A characteristic phase portrait for the situation when both cycles $P_{2}$ and $P_{4}$ have already become stable is presented in Fig. 3. This figure corresponds 


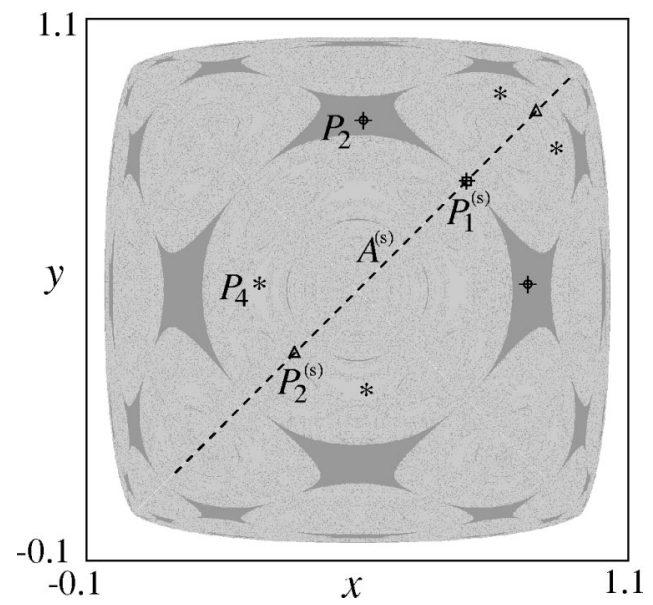

FIG. 3. Phase portrait of the globally coupled map system (1) reduced to the symmetric two-cluster subspace $[p=0.5$ in Eq. (3) after stabilization of both the asynchronous period-2 (denoted by $P_{2}$ and plotted by crossed circles) and the asynchronous period-4 (denoted by $P_{4}$ and plotted by stars) cycles. After the blowout bifurcation, the coherent state $A^{(s)}$ (dashed line segment) is a chaotic saddle. The symmetric fixed point (denoted by $P_{1}^{(s)}$ and plotted by the crossed square) and symmetric period- 2 cycle (denoted by $P_{2}^{(s)}$ and plotted by triangles) are repellors being after the transverse period-doubling bifurcations which give birth to $P_{2}$ and $P_{4}$. Basins of attraction for the cycles $P_{2}$ and $P_{4}$ are shown in dark and light grey, respectively. Parameters $a=3.9$ and $\varepsilon=0.345$. With further reduction of $\varepsilon$ each of the cycles $P_{2}$ and $P_{4}$ undergoes a sequence of additional bifurcations leading to various forms of quasiperiodic and chaotic two-cluster dynamics.

to a parameter point in the region $\mathfrak{R}_{2}$ of Fig. 1 where the synchronized state is a chaotic saddle.

For the case of slightly asymmetric clusters, the cycles $P_{2}$ and $P_{4}$ can be obtained by continuation of those in the symmetric case with the parameter $p$ (starting with $p=0.5$ ). If $p \neq 0.5$, these cycles stabilize in saddle-node bifurcations off the main diagonal rather than via inverse, subcritical pitchfork bifurcations as in the symmetric case $(p=0.5)$.

Figure 4 shows the regions of stability for the various types of dynamics that evolve from $P_{2}$ and $P_{4}$ under variation of $p$ and $\varepsilon$ for two different values of the nonlinearity parameter $a$. In Fig. 4(a) $(a=3.8)$, the upper boundary of the stability region (solid curve denoted $\mathrm{SN}$ ) defines the moment of stabilization of the asynchronous period-2 cycles $P_{2}$ in the afore-said saddle-node bifurcations. This curve is clearly seen to assume its maximal value for $p=0.5$, representing the fact that symmetric clusters will stabilize before slightly asymmetric clusters as $\varepsilon$ is reduced.

For $a=3.8$, stabilization of $P_{4}$ occurs at lower values of the coupling parameter than stabilization of $P_{2}$, and we find the stability region for $P_{4}$ (and for solutions developed from $P_{4}$ ) in the upper right corner of the stability region for $P_{2}$. For $a=4.0$ [Fig. 4(b)], on the other hand, $P_{4}$ stabilizes before $P_{2}$ (see Fig. 1), and the stability region for $P_{4}$ falls above that of $P_{2}$.

As shown in Sec. II, the stability of a periodic cycle in the two-cluster phase plane implies its stability in $\mathrm{N}$-dimensional phase space. Hence, the uppermost curves in Figs. 4(a) and
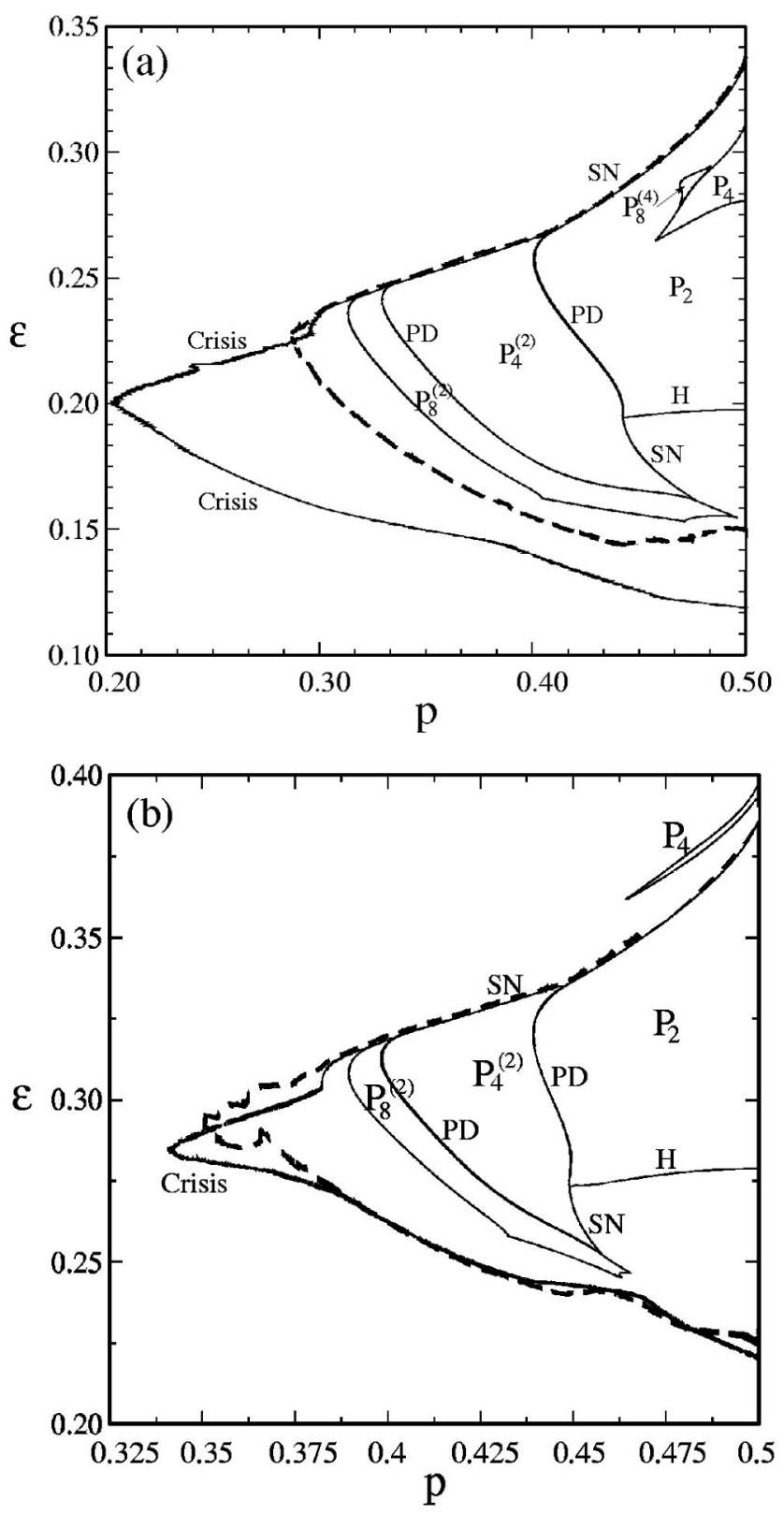

FIG. 4. Stability regions in the $(p, \varepsilon)$-parameter plane for the various types of dynamics in system (3) that develop from the asynchronous period-2 $\left(P_{2}\right)$ and period-4 $\left(P_{4}\right)$ cycles and represent two-cluster states in Eq. (1). Bifurcation curves denoted by SN, PD, and $H$ correspond to saddle-node, period-doubling, and Hopf bifurcations, respectively. With decreasing values of $p$ we can follow $P_{2}$ through a cascade of period-doubling bifurcations into a chaotic off-diagonal attractor that finally destroys in a boundary crisis. The bold dashed curve bounds the region where the largest Lyapunov exponent transverse to the two-cluster state is negative. Here, system (1) displays stable two-cluster states with a distribution between clusters as defined by $p$ and a dynamics that is given by the attractors developed from $P_{2}$. Parameters $a=3.8$ in (a) and $a=4$ in (b).

4(b) are the bifurcation curves in the $(p, \varepsilon)$-parameter plane for the appearance of the symmetric (or nearly symmetric) two-cluster states. The overlapping stability regions for $P_{2}$ and $P_{4}$ imply that the system has two coexisting types of 

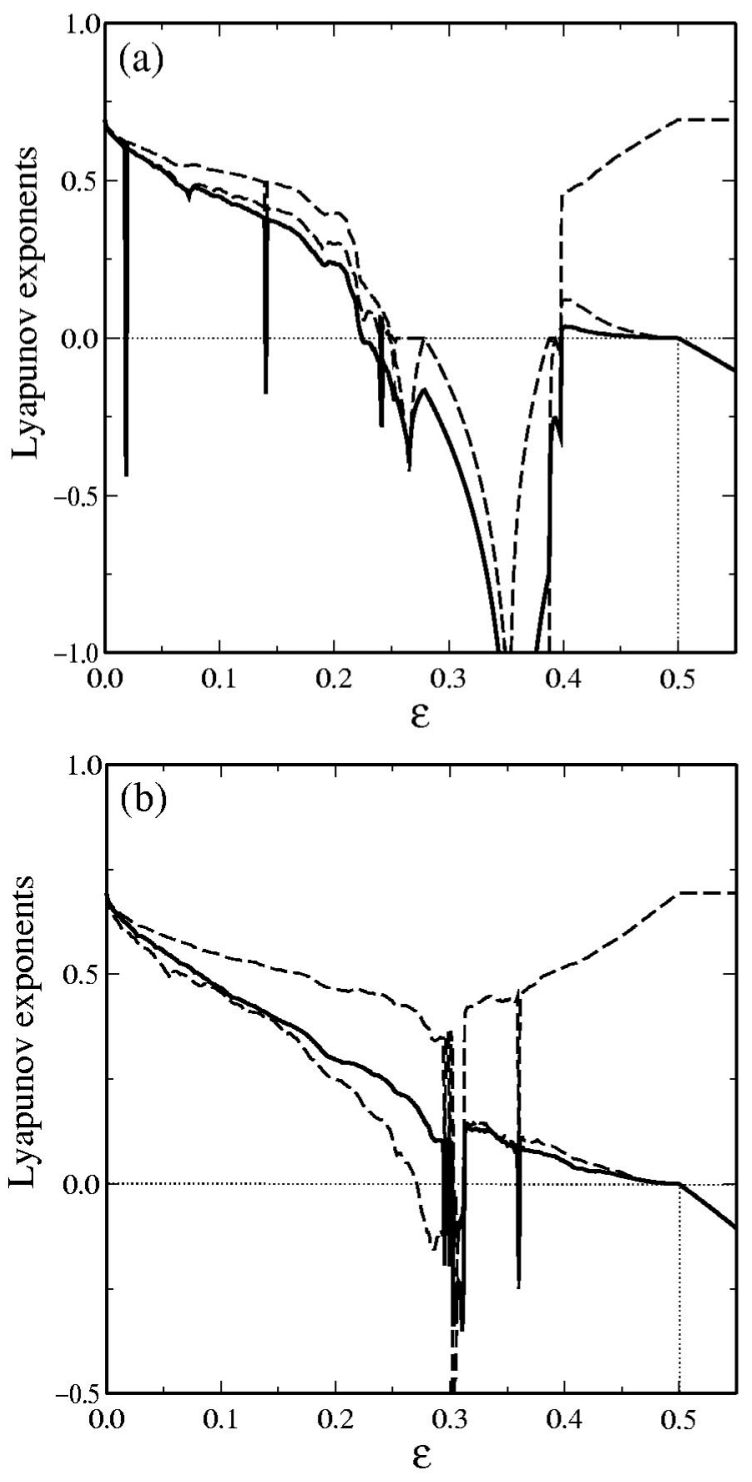

FIG. 5. Variation of the largest transverse Lyapunov exponent (solid bold curve) with the coupling parameter $\varepsilon$ for the two-cluster states being (a) symmetric $(p=0.5)$ or (b) with $2: 1$ variable distribution $(p=1 / 3)$. Parameter $a=4$. Dashed curves represent Lyapunov exponents within the two-cluster state. Note that, when $\varepsilon$ decreases, the state stabilizes in $N$ dimensions if it becomes an attracting cycle. Our interest is focused on the behavior immediately after the blowout bifurcation of the coherent state, which occurs at $\varepsilon=0.5$.

two-cluster dynamics (see, e.g., Fig. 3). With further variation of the parameters $p$ and $\varepsilon$, the cycles $P_{2}$ and $P_{4}$ undergo a variety of different bifurcations in which more complicated two-cluster dynamics arises. Besides periodic cycles of higher periodicity, quasiperiodic and chaotic dynamics occur. Some of the bifurcation curves are indicated in Figs. 4(a) and 4(b) where period-doubling and Hopf bifurcation curves are denoted PD and $H$, respectively. A more detailed examination of these dynamics falls outside the scope of the present paper. We refer the reader to a previous study [20] where a number of results in this direction are presented.
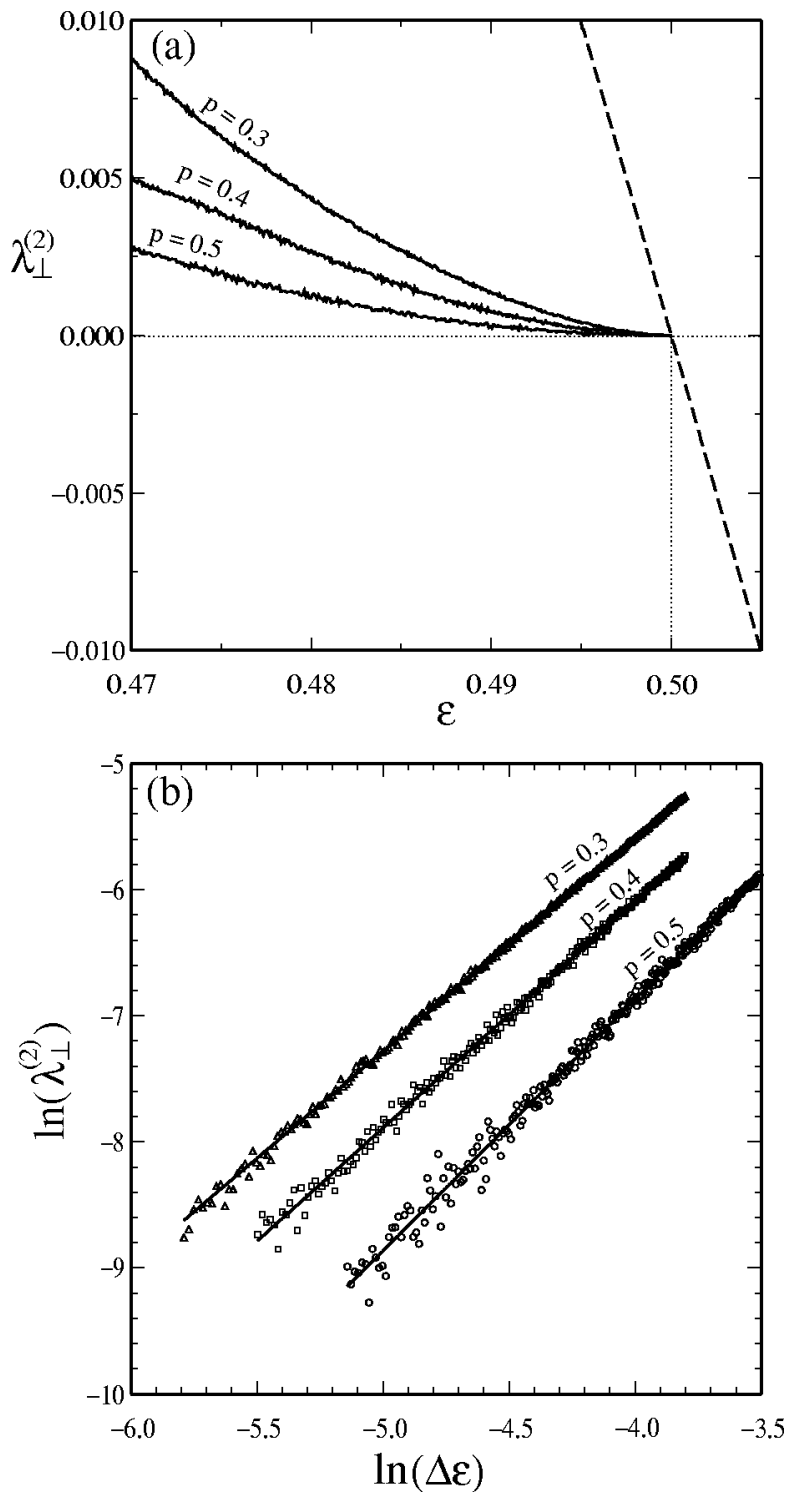

FIG. 6. Variation of the largest transverse Lyapunov exponent $\lambda_{\perp}^{(2)}$ for the chaotic two-cluster state $A^{(2)}$ arising immediately after the blowout bifurcation of the coherent state $A^{(s)}$. Here, $A^{(2)}$ has a form similar to those shown in Fig. 2(b), and the blowout bifurcation occurs at $\varepsilon=0.5$. Parameter $a=4$. The three curves in (a) represent different values of the asymmetry parameter $p$. In all cases, the transverse Lyapunov exponent is positive (although small). The dashed curve gives a variation of the transverse Lyapunov exponent $\lambda_{\perp}^{(1)}$ of the coherent state $A^{(s)}$. In (b), the same graphs in logarithmic scale illustrate the power law dependence (8). Here, the transverse Lyapunov exponents for $p=0.5, p=0.4$, and $p=0.3$ are plotted by circles, squares, and triangles, respectively. As one can see, straight lines within the marks fit the values of the exponents and have slopes $\alpha=2(p=0.5), \alpha=1.8 \quad(p=0.4)$, and $\alpha=1.7 \quad(p$ $=0.3$ ). We conclude that the chaotic two-cluster state formed in this process cannot be stable in $\mathrm{N}$-dimensional phase space.

If the attractor in a two-cluster state is quasiperiodic or chaotic, its stability within the two-cluster state does not imply its stability in the full $N$-dimensional phase space. The bold dashed curves in Figs. 4(a) and 4(b) denote the transverse destabilization of the two-cluster attractors developed 


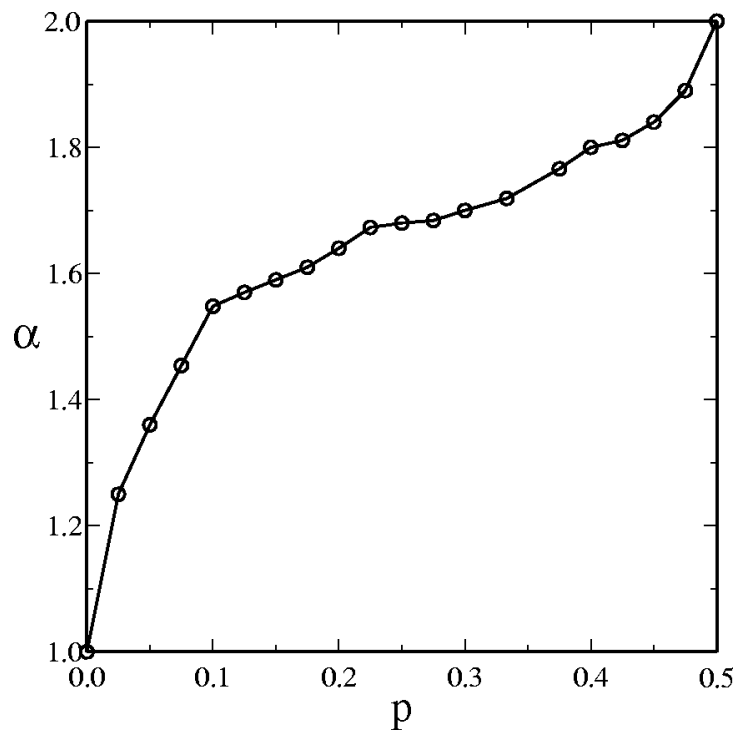

FIG. 7. Variation of the exponent $\alpha$ in the power law $\lambda_{\perp}^{(2)}$ $\sim\left|\varepsilon_{b l}-\varepsilon\right|^{\alpha}$ with the asymmetry parameter $p$. For symmetric clusters $(p=0.5), \alpha \approx 2$, and $\alpha \rightarrow 1$ as $p \rightarrow 0$. Parameter $a=4$.

from $P_{2}$, and the lower right curves represent their final boundary crises. The upper branch of the dashed curve coincides with the saddle-node bifurcation curve of two-cluster stabilization. As we can see, there is a fairly large parameter region where the attractor in the two-cluster state is quasiperiodic and yet transversely stable. Below this region there is another region where the two-cluster state is transversely unstable.

\section{TRANSVERSE INSTABILITY OF CHAOTIC CLUSTERS}

In this section we show that the chaotic motions in the two- and three-cluster states in general are transversely unstable. This applies in particular to the chaotic motions that appear after the blowout bifurcation of the coherent attractor $A^{(s)}$. To verify this, we show that the largest transverse Lyapunov exponents $\lambda_{\perp}^{(2)}$ (for the two-cluster state) and $\lambda_{\perp}^{(3)}$ (for the three-cluster state) are positive. Moreover, immediately after the blowout bifurcation $\varepsilon_{b l}$ they grow in accordance with a power law.

\section{A. Chaotic two-cluster state}

Figures 5(a) and 5(b) display scans of $\lambda_{\perp}^{(2)}$ over the range from $\varepsilon=0$ (uncoupled system) to right above the blowout bifurcation ( $\varepsilon=0.5$ ) for $a=4$ and for two different values of the asymmetry parameter: $p=0.5$ (symmetric clusters) and $p=1 / 3$ (1:2 cluster distribution). The scans of $\lambda_{\perp}^{(2)}$ are shown as bold curves. The dashed curves show the variation of the two Lyapunov exponents that control the two-dimensional cluster dynamics (3). In both cases the periodic two-cluster states stabilize after the blowout bifurcation and this gives rise to a hyperchaotic attractor bounded in two-dimensional phase space by the absorbing area $\mathcal{A}$ (see Fig. 2 where the characteristic form of $\mathcal{A}$ is illustrated). In Fig. 5(a) there is an interval around $\varepsilon=0.23$ where $\lambda_{\perp}^{(2)}$ is negative while the Lyapunov exponents in the two-cluster plane are positive. Here, we have a transversely stable chaotic two-cluster state. However, through most of the scan the transverse Lyapunov exponent is positive when the longitudinal exponents are positive.

Figure 6 shows an enlargement of the rightmost parts of the graphs from Fig. 5 in order to illustrate the power law of growth for $\lambda_{\perp}^{(2)}$ after the blowout bifurcation for different values of the asymmetry parameter $p$. Here, $a=4$. As we can see

$$
\lambda_{\perp}^{(2)} \sim\left|\varepsilon_{b l}-\varepsilon\right|^{\alpha}, \quad \varepsilon \rightarrow \varepsilon_{b l},
$$

where $\alpha \cong 2$ for the symmetric case $p=0.5$ and decreases with decreasing $p$. This result is supported by plotting the graphs on logarithmic scale [Fig. 6(b)]. Here, $\Delta \varepsilon=\varepsilon_{b l}-\varepsilon$, and the slopes of the linear part of the graphs determine the exponent $\alpha$ in the power law (8).

The graph of $\alpha=\alpha(p)$ as a function of $p$ is shown in Fig. 7. As it can be seen, $\alpha$ decreases with decreasing $p$. Moreover, $\alpha$ tends to 1 as $p$ approaches 0 and to 2 as $p$ approaches 0.5 .

\section{B. Chaotic three-cluster state}

As we have just shown, chaotic two-cluster motions, when they appear after destabilization of the coherent phase, are transversely unstable. It follows that the chaotic motions that arise must at least be three dimensional. We now show that the dimension must also be larger than three. To this end, we give a numerical evidence that chaotic motions in the symmetric three-cluster states are transversely unstable.

Figure 8 shows a plot of the transverse Lyapunov exponent $\lambda_{\perp}^{(3)}$ versus parameter $\varepsilon$ for a symmetric three-cluster state. $\lambda_{\perp}^{(3)}$ becomes positive immediately after the blowout bifurcation $(\varepsilon=0.5)$ and appears to grow in accordance with a power law similar to Eq. (8). This can be justified as follows. As illustrated in Fig. 9, the typical trajectory in the chaotic three-cluster state behaves in such a way that it spends most of the time very near the diagonal twodimensional planes $\sigma_{z}=\{x=y, z\}, \sigma_{y}=\{x=z, y\}$, and $\sigma_{x}$ $=\{x, y=z\}$. Moreover, it switches between these planes in an apparently random manner. From this observation we conclude that an approximate value for the transverse (to the three-cluster state) Lyapunov exponent $\lambda_{\perp}^{(3)}$ can be obtained as calculated on the planes $\sigma_{x}, \sigma_{y}$, and $\sigma_{z}$, with the additional assumption that the average time spent near each of these planes is the same. This gives

$$
\lambda_{\perp}^{(3)} \cong\left(2 \lambda_{\perp, 1}^{(2)}+\lambda_{\perp, 2}^{(2)}\right) / 3
$$

where $\lambda_{\perp, 1}^{(2)}$ and $\lambda_{\perp, 2}^{(2)}$ are the largest and the second transverse Lyapunov exponents for the chaotic motions in the twocluster planes $\sigma_{x}, \sigma_{y}$, and $\sigma_{z}$. Using the expression (6) for the transverse Lyapunov exponents for two-cluster states and the formula (7) for three-cluster states, we come to the approximate formula (9). 


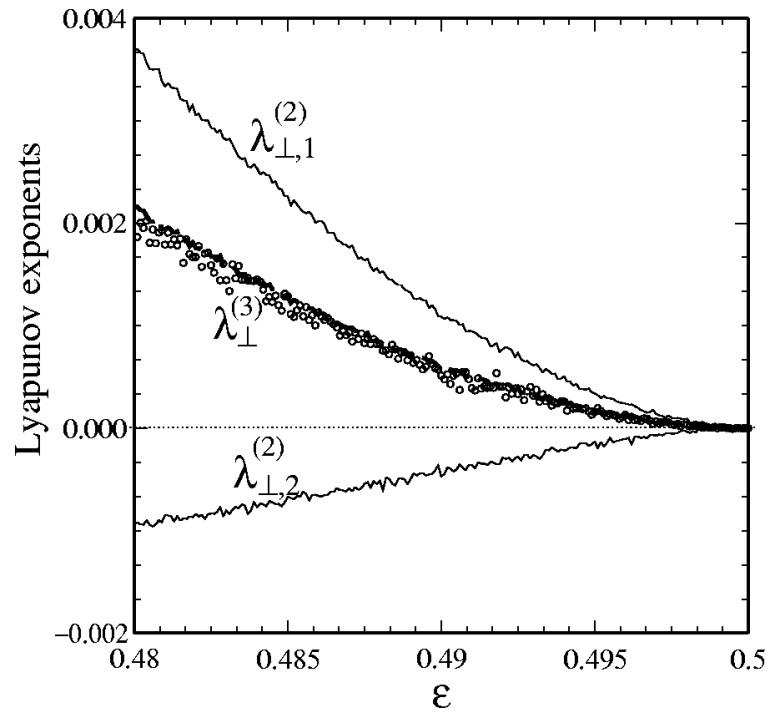

FIG. 8. Transverse Lyapunov exponent $\lambda_{\perp}^{(3)}$ (shown by circles) for a symmetric three-cluster state as a function of the coupling parameter $\varepsilon$. The largest $\lambda_{\perp, 1}^{(2)}$ and the second $\lambda_{\perp, 2}^{(2)}$ transverse Lyapunov exponents for the two-cluster state with $2: 1 \quad(p=1 / 3)$ variable distribution between clusters are also shown. The value $\left(2 \lambda_{\perp, 1}^{(2)}+\lambda_{\perp, 2}^{(2)}\right) / 3$ is represented by the bold dashed curve that fits the values of $\lambda_{\perp}^{(3)}$. We conclude that $\lambda_{\perp}^{(3)}$ becomes positive immediately after the blowout bifurcation $(\varepsilon=0.5)$ and grows in accordance with a power law. Here, $a=4$.

To conclude our considerations, we note that the numerical calculation of $\lambda_{\perp}^{(3)}$ has required the introduction of small noise of the order of $10^{-22}$. Without this noise, trajectories are captured by the two-cluster dynamics because of final precision in the calculations. The average capturing times are shown in Fig. 10 for single $\left(10^{-8}\right)$, double $\left(10^{-16}\right)$, and triple $\left(10^{-24}\right)$ precisions, respectively. We suppose that this capturing phenomenon can explain why high-dimensional chaotic motions arising after desynchronization of the coherent phase have not previously been reported. Indeed, any regular calculation (without noise) gives evidence of twocluster dynamics even though this is actually transversely unstable as soon as it is chaotic.

It was shown in [21] that cluster states arising after blowout bifurcation of a coherent attractor [see Fig. 2(b)] cannot be asymptotically stable in the whole $N$-dimensional phase space of the globally coupled map system (1). As we can see now, the lack of a parameter region of asymptotic stability may cause these clusters states to also be unstable on the average.

\section{CONCLUSION}

In order to refine the original phase diagram presented by Kaneko [1] we investigated in detail the bifurcations involved in the loss of complete synchronization and the formation of clusters of partially synchronized oscillators in a system of globally coupled logistic maps. We found that the loss of complete synchronization and the formation of stable two-cluster states are two distinct processes. Moreover, depending sensitively on the nonlinearity parameter $a$ of the
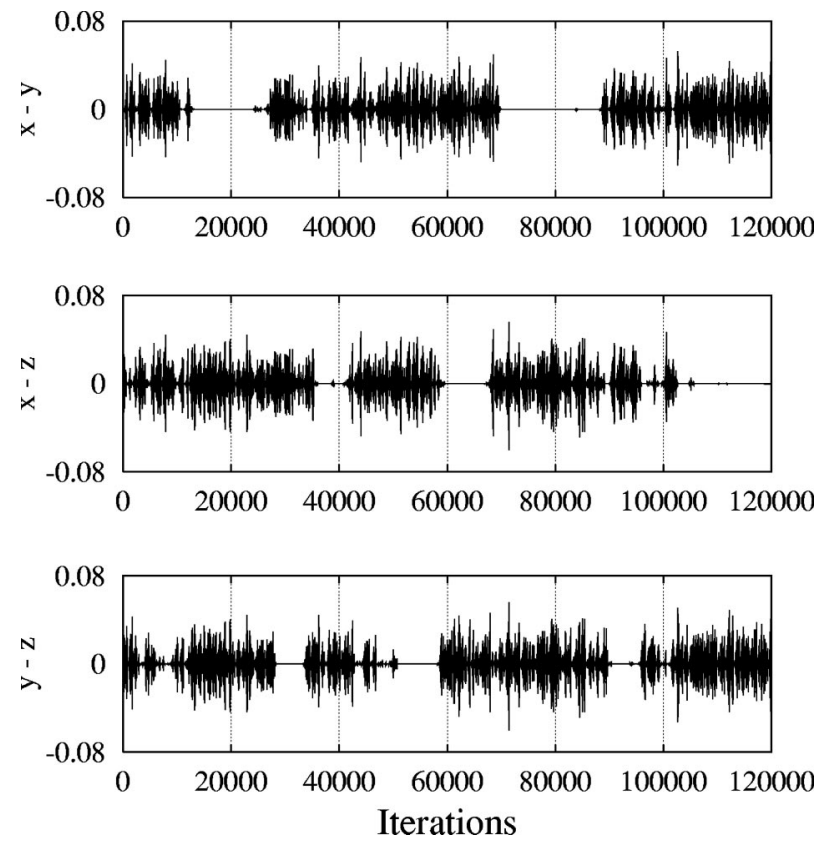

FIG. 9. Synchronization errors calculated on a typical trajectory for the chaotic three-cluster state [considering system Eq. (5) with $K=3$ and $\left.\left.p_{j}=1 / 3, j=1,2,3\right)\right]$. We have added a small noise of the maximal amplitude $10^{-22}$. The first $10^{4}$ iterations are skipped, and the next $1.2 \times 10^{5}$ iterations are plotted. The trajectory spends most of its time near the two-dimensional planes $\sigma_{z}=\{x=y, z\}, \sigma_{y}=\{x$ $=z, y\}$ and $\sigma_{x}=\{x, y=z\}$, and it switches between these planes in an apparently random manner. Parameters $a=4$ and $\varepsilon=0.495$.

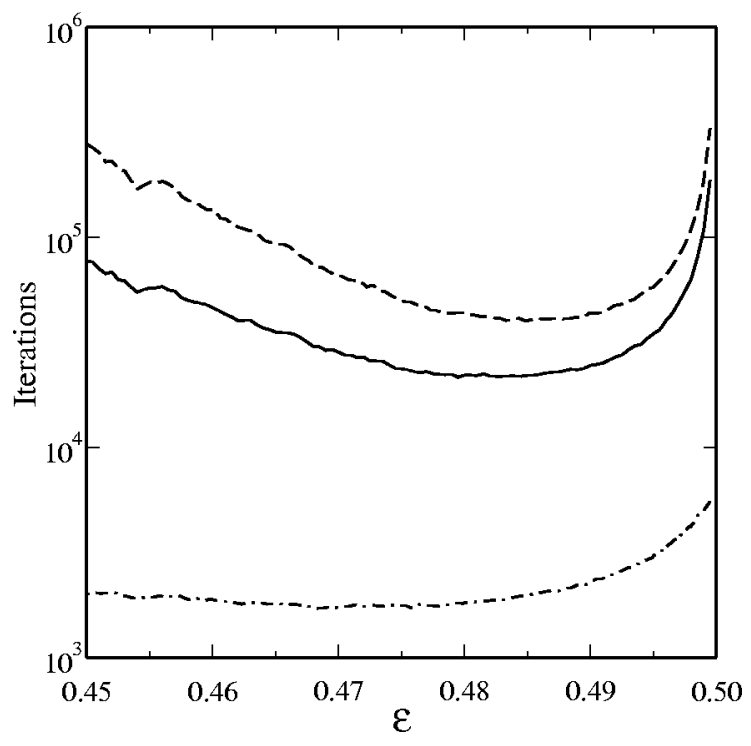

FIG. 10. Average (over 8000 initial conditions) capturing time in a two-cluster state as calculated with single $\left(10^{-8}\right)$, double $\left(10^{-16}\right)$, and triple $\left(10^{-24}\right)$ precisions and shown by dotted-dashed, solid, and dashed curves, respectively. By iterating system (5) with $K=3, p_{j}=1 / 3, j=1,2,3$, and $a=4$, we find that all trajectories are captured by transversally unstable two-cluster states in a finite time. The capturing phenomenon and the associated spurious stability with positive transverse Lyapunov exponents can be avoided by adding a small amount of noise to the numerical computations. 
logistic map, the stabilization of symmetric (or nearly symmetric) two-cluster states may occur before or after the blowout bifurcation, leading to very different behaviors.

The loss of complete synchronization proceeds via a riddling bifurcation (in which the fixed point loses its transverse stability in a period-doubling bifurcation) and a blowout bifurcation (in which the transverse Lyapunov exponent for a typical trajectory on the synchronized chaotic set becomes positive). If the blowout bifurcation occurs before the formation of stable two-cluster dynamics becomes possible, the synchronized chaotic state explodes into a high-dimensional hyperchaotic state. The reason why this type of transition has not previously been observed appears to be related to spurious numerical effects that cause the computed trajectories to be captured in low-dimensional chaotic states, even though these states are transversely unstable. We showed that chaotic two- and three-cluster states are transversely unstable immediately after the blowout bifurcation and obtained the scaling relations relating to the variation of the maximal transverse Lyapunov exponent with the distance to the bifurcation point.

Formation of the two-cluster states takes place via the stabilization of asynchronous periodic cycles. For coupled logistic maps (with the considered coupling structure) only the asynchronous period- 2 and period- 4 cycles are involved in the initial stage of the formation of the symmetric twocluster state. We proved that if any of these cycles are stable in the phase space of the symmetric two-cluster state, then they are also stable in the whole $N$-dimensional phase space.
We also considered the influence of a small asymmetry in the distribution of oscillators between the clusters of the twocluster state. While stabilization of the symmetric two-cluster state takes place via an inverse, subcritical pitchfork bifurcation, stabilization of asymmetric two-cluster states occurs via a saddle-node bifurcation off the main diagonal. With further reduction of the coupling parameter, the asynchronous cycles forming the two-cluster state may undergo additional bifurcations in which more complex behaviors arise. Chaotic twocluster dynamics may occur in relatively small regions of parameter space when the two-cluster attractor lies away from the main diagonal. In the phase space of the two-cluster state, this type of behavior continues to be attracting until it is destroyed in a boundary crisis. In the full $\mathrm{N}$-dimensional phase space, however, the chaotic two-cluster dynamics tends to be transversely unstable.

We described a simple general algorithm of finding stable cluster states based on the form of transverse Lyapunov exponents. Considering only a low-dimensional reduced system of coupled maps and calculating the corresponding transverse Lyapunov exponents, one can evaluate the stability of the cluster states for a very large lattice of globally coupled maps.

\section{ACKNOWLEDGMENT}

We thank A. Pikovsky for a number of illuminating discussions.
[1] K. Kaneko, Physica D 41, 137 (1990).

[2] K. Kaneko, Physica D 75, 55 (1994).

[3] A. Sherman and J. Rinzel, Biophys. J. 59, 547 (1991).

[4] J. Sturis, E. Van Cauter, J. D. Blackman, and K. S. Polonsky, J. Clin. Invest. 87, 439 (1991).

[5] E. Mosekilde, Topics in Nonlinear Dynamics, Applications to Physics, Biology and Economic Systems (World Scientific, Singapore, 1996).

[6] K. Wiesenfeld and P. Hadley, Phys. Rev. Lett. 62, 1335 (1989).

[7] K. Wiesenfeld, C. Bracikowski, G. James, and R. Roy, Phys. Rev. Lett. 65, 1749 (1990).

[8] W. Wang, I. Z. Kiss, and J. L. Hudson, Chaos 10, 248 (2000).

[9] K. Kaneko, Physica D 103, 505 (1997).

[10] K. Kaneko, Phys. Rev. Lett. 78, 2736 (1997); Physica D 124, 322 (1998).

[11] J. Milnor, Commun. Math. Phys. 99, 117 (1985).

[12] J. C. Alexander, J. A. Yorke, Z. You, and I. Kan, Int. J. Bifurcation Chaos Appl. Sci. Eng. 2, 795 (1992).

[13] J. C. Sommerer and E. Ott, Nature (London) 365, 136 (1993); E. Ott and J. C. Sommerer, Phys. Lett. A 188, 39 (1994).

[14] N. Platt, E. A. Spiegel, and C. Tresser, Phys. Rev. Lett. 70, 279 (1993).

[15] F. Xie and G. Hu, Phys. Rev. E 56, 1567 (1997).

[16] P. Glendinning, Phys. Lett. A 259, 129 (1999); 264, 303 (1999).

[17] Yu. L. Maistrenko, V. L. Maistrenko, A. Popovich, and E.
Mosekilde, Phys. Rev. E 57, 2713 (1998); 60, 2817 (1999).

[18] P. Ashwin, J. Buescu, and I. Stewart, Phys. Lett. A 193, 126 (1994); Nonlinearity 9, 703 (1996).

[19] Y.-C. Lai, C. Grebogi, J. A. Yorke, and S. C. Venkataramani, Phys. Rev. Lett. 77, 55 (1996).

[20] A. Popovich, Yu. Maistrenko, E. Moseskilde, A. Pikovsky, and J. Kurths, Phys. Lett. A 275, 401 (2000).

[21] Yu. Maistrenko, A. Popovich, and M. Hasler, Int. J. Bifurcation Chaos Appl. Sci. Eng. 10, 179 (2000).

[22] A. V. Taborov, Yu. L. Maistrenko, and E. Mosekilde, Int. J. Bifurcation Chaos Appl. Sci. Eng. 10, 1051 (2000).

[23] A. Hamm, Physica D 142, 41 (2000).

[24] N. B. Ouchi and K. Kaneko, Chaos 10, 359 (2000).

[25] V. Belykh, I. Belykh, N. Komrakov, and E. Mosekilde, Discrete Dyn. Nat. Soc. 4, 245 (2000).

[26] H. Chaté and P. Manneville, Europhys. Lett. 17, 291 (1992); Prog. Theor. Phys. 87, 1 (1992).

[27] J. W. Shuai, K. W. Wong, and L. M. Cheng, Phys. Rev. E 56, 2272 (1997).

[28] C. Zhou and C.-H. Lai, Physica D 135, 1 (2000).

[29] C. Mira, L. Gardini, A. Barugola, and J.-C. Cathala, Chaotic Dynamics in Two-Dimensional Noninvertible Maps (World Scientific, Singapore, 1996).

[30] Yu. L. Maistrenko, V. L. Maistrenko, A. Popovich, and E. Mosekilde, Phys. Rev. Lett. 80, 1638 (1998).

[31] A. Popovich, Yu. Maistrenko, and E. Mosekilde (unpublished). 